\title{
Communication
}

\section{Automatic and Accurate Sleep Stage Classification via a Convolutional Deep Neural Network and Nanomembrane Electrodes}

\author{
Kangkyu Kwon ${ }^{1,2,+} \mathbb{D}$, Shinjae Kwon ${ }^{2,3,+}$ and Woon-Hong Yeo ${ }^{2,3,4,5, *(\mathbb{D})}$ \\ 1 School of Electrical and Computer Engineering, Georgia Institute of Technology, Atlanta, GA 30332, USA; \\ kkwon49@gatech.edu \\ 2 IEN Center for Human-Centric Interfaces and Engineering, Institute for Electronics and Nanotechnology, \\ Georgia Institute of Technology, Atlanta, GA 30332, USA; skwon64@gatech.edu \\ 3 George W. Woodruff School of Mechanical Engineering, Georgia Institute of Technology, \\ Atlanta, GA 30332, USA \\ 4 Wallace H. Coulter Department of Biomedical Engineering, Parker H. Petit Institute for Bioengineering and \\ Biosciences, Georgia Institute of Technology, Atlanta, GA 30332, USA \\ 5 Neural Engineering Center, Institute for Materials, Institute for Robotics and Intelligent Machines, \\ Georgia Institute of Technology, Atlanta, GA 30332, USA \\ * Correspondence: whyeo@gatech.edu; Tel.: +1-404-385-5710; Fax: +1-404-894-1658 \\ + These authors contributed equally to this work.
}

check for updates

Citation: Kwon, K.; Kwon, S.; Yeo, W.-H. Automatic and Accurate Sleep Stage Classification via a Convolutional Deep Neural Network and Nanomembrane Electrodes. Biosensors 2022, 12, 155. https:// doi.org/10.3390/bios12030155

Received: 13 December 2021 Accepted: 28 February 2022 Published: 2 March 2022

Publisher's Note: MDPI stays neutral with regard to jurisdictional claims in published maps and institutional affiliations.

Copyright: (C) 2022 by the authors. Licensee MDPI, Basel, Switzerland. This article is an open access article distributed under the terms and conditions of the Creative Commons Attribution (CC BY) license (https:// creativecommons.org/licenses/by/ $4.0 /)$.
Abstract: Sleep stage classification is an essential process of diagnosing sleep disorders and related diseases. Automatic sleep stage classification using machine learning has been widely studied due to its higher efficiency compared with manual scoring. Typically, a few polysomnography data are selected as input signals, and human experts label the corresponding sleep stages manually. However, the manual process includes human error and inconsistency in the scoring and stage classification. Here, we present a convolutional neural network (CNN)-based classification method that offers highly accurate, automatic sleep stage detection, validated by a public dataset and new data measured by wearable nanomembrane dry electrodes. First, our study makes a training and validation model using a public dataset with two brain signal and two eye signal channels. Then, we validate this model with a new dataset measured by a set of nanomembrane electrodes. The result of the automatic sleep stage classification shows that our CNN model with multi-taper spectrogram pre-processing achieved $88.85 \%$ training accuracy on the validation dataset and $81.52 \%$ prediction accuracy on our laboratory dataset. These results validate the reliability of our classification method on the standard polysomnography dataset and the transferability of our CNN model for other datasets measured with the wearable electrodes.

Keywords: automatic sleep stage classification; convolutional neural network; nanomembrane electrode; multi-taper spectrogram

\section{Introduction}

An accurate sleep stage classification [1-3] plays a significant role in sleep quality monitoring and the diagnosis of disorders. The polysomnogram (PSG) is widely used in the diagnosis of obstructive sleep apnea (OSA) syndrome [4]. PSG is non-invasive and consists of a simultaneous recording of multiple physiological parameters related to sleep and sleep disorders. Standard polysomnography includes the measurement of various physiological signals such as an electroencephalogram (EEG), an electrooculogram (EOG), an electromyogram (EMG), and an electrocardiogram (ECG). Typically, a series of polysomnographic signals for a 30-second-long epoch is labeled as a certain sleep stage by an expert sleep scorer. 
Compared to the manual scoring of sleep stages, automatic sleep stage classification serves as a more efficient way to evaluate a large amount of sleep data. Machine learning algorithms have been adopted in automatic sleep stage classification to increase classification efficiency and performance in recent years. Among them, conventional statistical machine learning algorithms, such as Support Vector Machine [1,5], Hidden Markov Model [6], k-nearest neighbors [7], and Random Forests [7] are adopted at the early stage. Recent progress of deep learning in computer vision, natural language processing, and robotics has advanced these methods to application in automatic sleep stage classification. A convolutional neural network (CNN) has been frequently employed for the task. The weight sharing mechanism at the convolutional layers forces the shift-invariance of the learned features and greatly reduces the model's complexity, consequently improving the model's generalization. Other network variants, such as Deep Belief Networks (DBNs), Auto-encoder, and Deep Neural Networks (DNNs), have also been explored. Moreover, Recurrent Neural Networks (RNNs), e.g., Long Short-Term Memory (LSTM), capable of sequential modeling, have been found to be efficient in capturing long-term sleep stage transitions. However, these methods still have limited accuracy in sleep stage classification as they have been trained and tested merely on a public dataset, without validation study with real lab datasets.

This work presents an automatic sleep stage classification model that could be applied to the public dataset, and that could also be applied to the classification of our laboratory datasets measured with a novel wearable system. In the public dataset from ISRUC used for this study [8], four channels of signals (two EEG and two EOG) were selected based on their proximity to the electrode locations of our new wearable system being tested. The four signals were preprocessed with various filters and a multi-taper spectral analysis was performed. They were then split into 30-second-long epochs and converted into spectrogram images to be used and tested with our newly developed CNN sleep stage classification model. This $\mathrm{CNN}$ model trained with a public dataset was then applied to our lab dataset measured with nanomembrane electrodes that were pre-processed with the same methods. A comparison study with a multi-taper spectrogram and band-pass-filtered raw signals showed the advantage of a multi-taper spectrogram in enhancing the transferability of the model. The final result of this study supports not only the performance of our new classification model on the standard PSG dataset, but also the model's transferability to a dataset measured with our novel system.

\section{Materials and Methods}

\subsection{ISRUC Public Dataset}

The ISRUC sleep dataset (Figure 1A) consists of complete overnight standard PSG recordings of 118 subjects with three health statuses (healthy, sick, and under-treatment). For this study, a total of 100 data from 100 subjects from subgroup 1 were used. ISRUC subgroup 1 included subjects aged between 20 and 85 years (51 on average), with 55 males and 45 females. The subjects were diagnosed with various sleep disorders. More details of individual subject information can be found in Khalighi et al. [8]. Each recording contained six EEG channels (i.e., C3-A2, C4-A1, F3-A2, O1-A2, O2-A1, and F4-A1), two EOG channels (i.e., LOC-A2 and ROC-A1), and three EMG channels (i.e., X1, X2, and X3) as well as an annotation file with detailed events. For this study, two of the EEG channels (F3-A2 and F4-A1) and both of the EOG channels (LOC-A2 and ROC-A1) were used. The recording rate was $200 \mathrm{~Hz}$. In addition, each 30-second-long epoch was labeled with one of the five sleep stages (W, N1, N2, N3, R), as scored by two experts according to the American Academy of Sleep Medicine (AASM) rules [8]. 
A

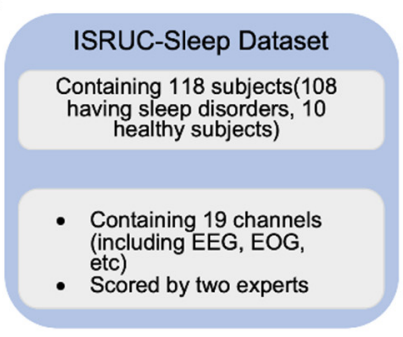

B

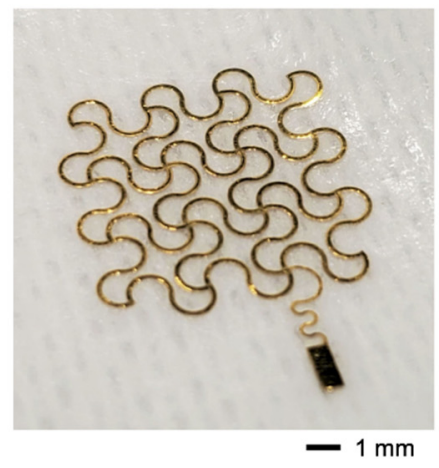

C

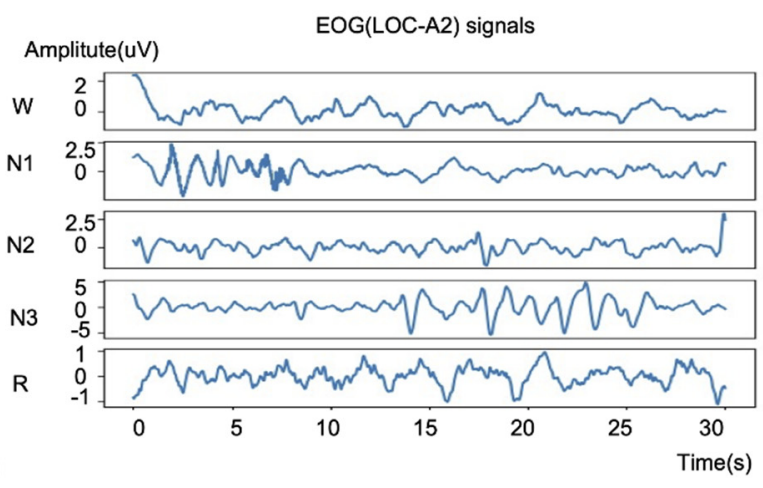

Containing 2 EOG channels, 2 EEG

Scored by one expert

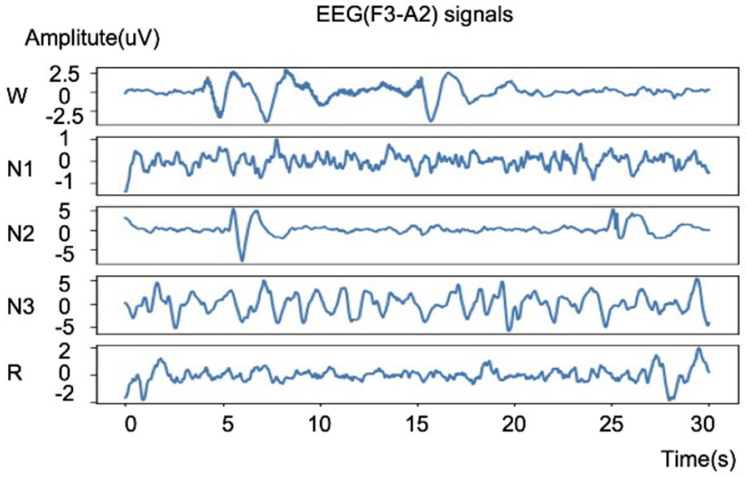

E

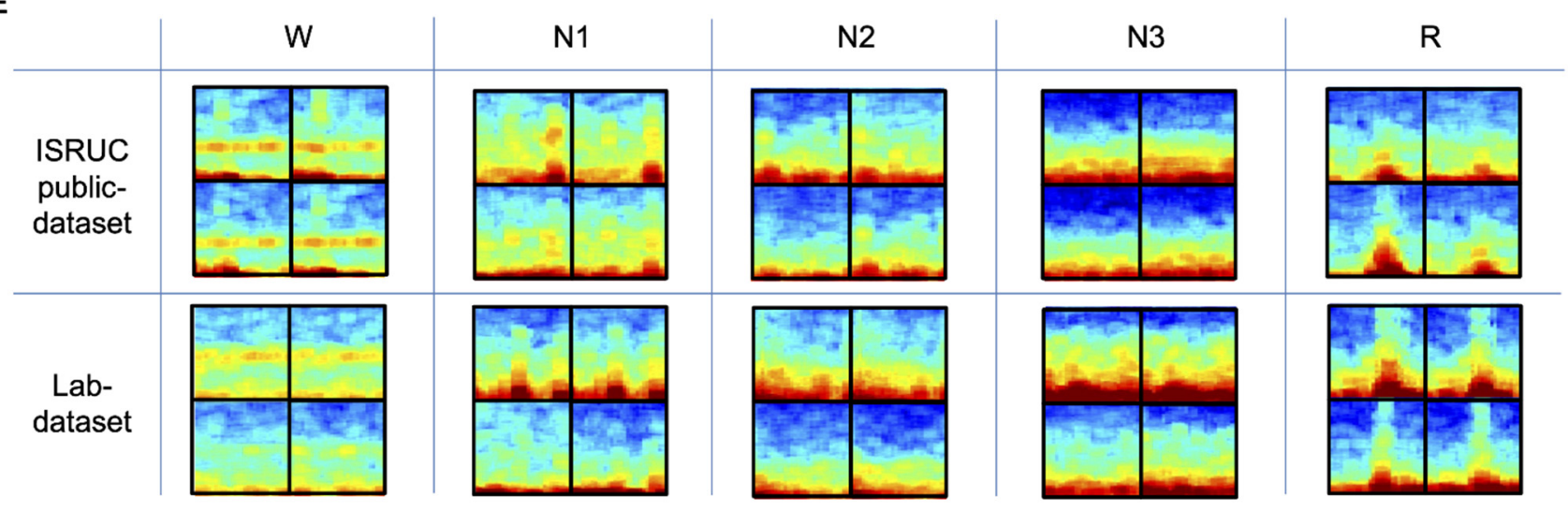

Figure 1. Overview of a public dataset (ISRUC) and the measured lab dataset. (A) Detailed information of both datasets. (B) Data recording system using nanomembrane bioelectrodes (left) and the sensor mounting locations (right) were the upper center of the forehead (EEG1 and EEG2) to measure two-channel EEG, one electrode on the lower-left corner of the left eye (EOG1) to measure two-channel EOG, and another electrode on the upper-right corner of the right eye (EOG2). (C) Measured EOG signals of five different sleep stages: W, N1, N2, N3, and R. (D) Measured EEG signals of five different sleep stages: W, N1, N2, N3, and R. (E) Examples of multi-taper spectrograms of both the ISRUC public and the lab datasets with five sleep stages. From top-to-bottom and left-to-right, the spectrograms show channels F3-A2, F4-A1, EOGL, and EOGR of the public dataset, and channels EEG1, EEG2, EOG1, and EOG2 of the lab dataset.

\subsection{Measured Lab Dataset}

The lab dataset used for this study was measured from four healthy male subjects aged between 24 and 27 years. A total of four data were collected, with one recording per subject. To measure our own dataset in the lab, we fabricated a set of nanomembrane, stretchable electrodes using a gold and polyimide (PI) composite, laminated on a silicone adhesive (Figure 1B). This fabrication utilized a microfabrication technique, including photolithography, developing, and etching for making stretchable patterns $[9,10]$. Afterward, 
the patterned electrode was transfer-printed onto a soft silicone elastomer for skin mounting [11,12]. For the two-channel EEG setup, two electrodes were placed near the upper center of the forehead (EEG1 and EEG2) to measure frontopolar EEG. For the two-channel EOG, one electrode was placed on the lower-left corner of the left eye (EOG1), and the other electrode was placed on the upper right corner of the right eye (EOG2). All the EEG and EOG channels were derived with a single common reference electrode placed on the bony area of the nose. In PSG, the mastoid is the most widely used common reference point, but the nose is also considered one of the inactive areas suitable as a common reference alternative to the mastoid. Some of the previous works related to EEG measurement adopted the nose as the reference on special occasions [13-16]. The nanomembrane dry electrode needs to be placed on clean skin without hair to measure signals with high quality, so the nose was selected as the common reference for this study. The ground electrode was placed on the forehead next to the EEG1 electrode. These electrode locations were chosen to develop a compact facial patch device for sleep monitoring in future studies.

A customized printed circuit board (PCB) with an nRF52 (Nordic Semiconductor, Trondheim, Norway) and an ADS 1299 (Texas Instruments, Dallas, TX, USA) was used to collect EEG and EOG signals at a sampling rate of $250 \mathrm{~Hz}$ and to transmit them to an Android mobile device via Bluetooth for data storage. The systems used to collect the lab datasets, the nanomembrane-based electrodes and the custom PCB with the nRF52 and ADS 1299 have been extensively studied and validated by comparison with wellestablished measurement systems by numerous related previous studies [17-21]. A set of example datasets in Figure 1C,D, measured by standard PSG setup and the wearable device, show EOG signals and EEG signals with different patterns based on sleep stages. Figure 1E shows representative multi-taper spectrograms of data measured by the standard PSG setup and the setup used for this study at each of the five sleep stages.

\subsection{Data Pre-Processing}

Rather than training an automatic classification model based on raw sleep data, the data pre-processing method was applied for better classification performance. In the ISRUC public dataset, preprocessing was already applied to eliminate undesired noise and DC offset, enhancing the signal quality and the signal-to-noise ratio. The filtering stage consisted of a notch filter to eliminate the $50 \mathrm{~Hz}$ powerline noise and a bandpass Butterworth filter with a lower cutoff of $0.3 \mathrm{~Hz}$ and a higher cutoff of $35 \mathrm{~Hz}$ for both the EEG and EOG channels. To maximize the performance and transferability of the classification model, the same bandpass filtering parameters were used for the lab dataset. The notch filter setting was adjusted to remove a $60 \mathrm{~Hz}$, rather than $50 \mathrm{~Hz}$, power line noise because the frequency of powerline noise varies based on the place of measurement. Moreover, to match the per-epoch data size to the public dataset, the lab dataset was down-sampled from $250 \mathrm{~Hz}$ to $200 \mathrm{~Hz}$ by interpolating the datapoints that matched the timepoints corresponding with the $200 \mathrm{~Hz}$ sampling rate.

\subsection{Input Dataset for Deep Learning}

EEG and EOG signals are commonly analyzed with time-frequency processing techniques or spectrograms, since they are frequently related to behavioral patterns [22]. CNN models are mainly applied to classify and recognize two-dimensional images due to their good compatibility and unprecedented ability to extract image features. As a result, there were many attempts to use spectrograms generated from EEG and EOG signals as the input dataset of a CNN [23-26]. The spectrogram used for this study was generated by multitaper spectral analysis, which utilizes multiple taper functions to compute single-taper spectra for better resolution and reduced bias and variance compared to the traditional method. The default settings and parameters provided by Prerau et al. were used for generating spectrograms [27]. The frequency range of spectral analysis was set between 0 and $20 \mathrm{~Hz}$. The time-half-bandwidth product was set to 5, and the number of tapers was set to 9 . A window size of $5 \mathrm{~s}$ was used, with a step size of $1 \mathrm{~s}$. The entire dataset 
was first converted into a multi-taper spectrogram, which was then segmented into a 30 -second-long epoch. The size of the spectrogram matrix was $30 \times 103$ for each epoch. Since four data channels were used for this study, four spectrogram matrices were put together as a $60 \times 206$ matrix, which was then converted into a PNG image file sized $256 \times 256$ (Figure 2A). The same dataset was prepared with raw data for a comparison study to show the advantage of using a multi-taper spectrogram. The dataset with raw data was composed of bandpass-filtered raw data. The filtered data were then segmented into 30-second-long epochs of four channels, with a matrix size of $4 \times 6000$. Figure $2 \mathrm{~B}$ is the flow diagram of how our training dataset (sampled from the ISRUC dataset) and testing dataset (sampled from the lab dataset) were processed.

i. Raw Signal

unununum

unumunum

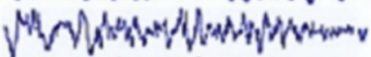

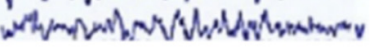

ii. Multi-Taper Spectrogram

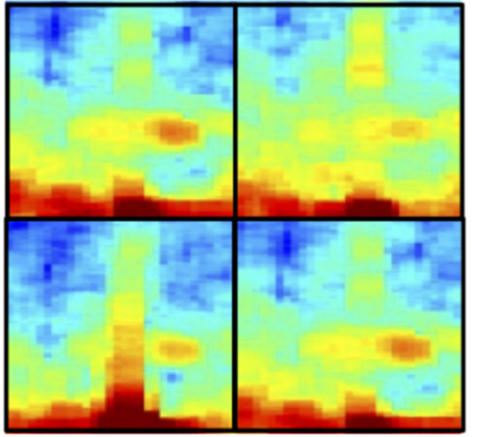

B

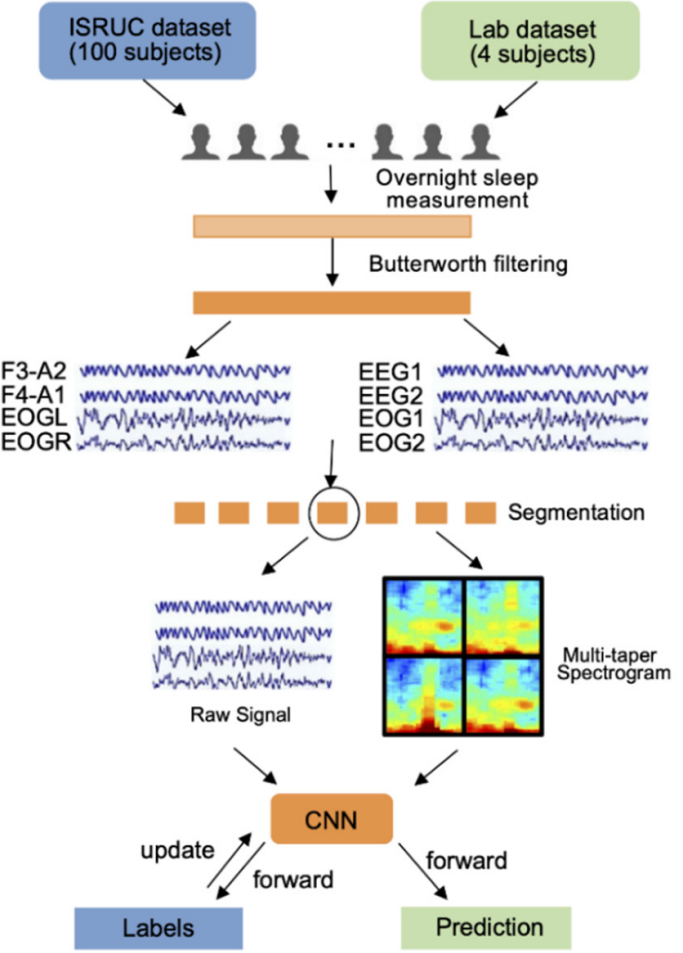

C

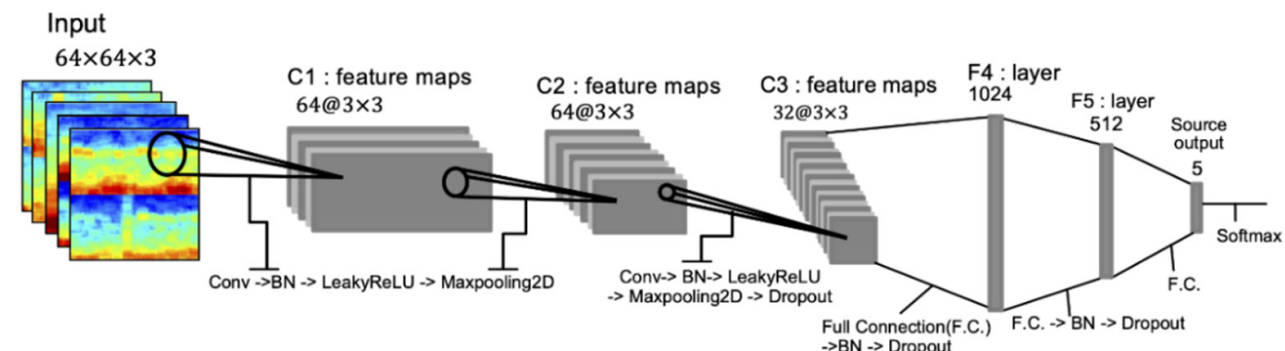

D

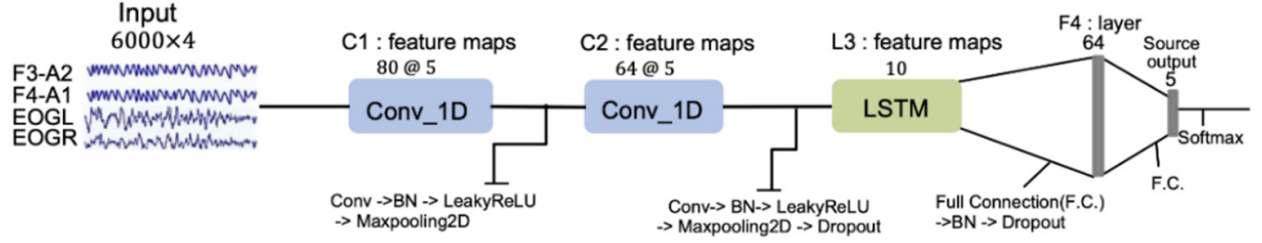

Figure 2. (A) Arrangement of four multi-taper spectrograms for deep learning dataset input. (B) Flow chart capturing data processing overview. (C,D) Proposed machine learning architectures for multitaper spectrograms (C) and raw signals (D); in this figure, Conv: convolution, F.C.: fully connected layers, and $\mathrm{BN}$ : batch normalization. 


\subsection{CNN-Based Classifier}

The architecture was developed by trial and error, drawing influence from earlier models $[28,29]$. Two models were created according to the form of input data: the CNN architecture in the case of image-based multi-taper spectrograms and the CNN + LSTM architecture in the case of time-based raw signal data. The sleep stage classification CNN architecture is described in Figure 2C,D. For the multi-taper spectrogram, the inputs of our CNN were 30 -second-long spectrogram images ( $256 \times 256$ pixels) of 4 channels (two EEG and two EOG) connected together in a square. The spectrogram image was then resized to $64 \times 64$ and converted to a value between $0-1$ using normalization. Since the color-image consisted of 3 channels (Red, Green, and Blue), every input matrix became a 3-dimensional matrix $(64 \times 64 \times 3)$. The non-linear activation function employed was the Leaky Rectified Linear Unit (Leaky ReLU). ADAM (learning rate $=0.002$ ) was utilized for the optimization of the $\mathrm{CNN}$ architecture. The batch size was set to 16 and the dropout deactivation rate was set to 0.5 . Early stopping was used to prevent overfitting by randomly eliminating $20 \%$ of the data from the training set and utilizing it as a validation set at the start of the optimization phase. When the validation loss stopped improving, learning rate annealing was performed with a factor of 5 . The training was terminated when two successive decays occurred with no network performance improvement on the validation set. A single convolutional cell (Conv_N) consisted of a convolutional layer, one layer of batch normalization, one layer of max pooling, and one layer of the Leaky ReLu function. The final output of the Deep Neural Network was a $5 \times 1$ vector. It was then passed through a softmax layer and finally outputted the predicted class (one of the five sleep stages). For the raw signals, the inputs of our CNN_LSTM were 30-second raw signal data of 4 channels (two EEG and two EOG) with an input size of $6000 \times 4$. The kernel layer was composed of two convolutional cells and one LSTM cell. Most of the set-ups were the same as those of the spectrogram $\mathrm{CNN}$ architecture, except structure, while the learning rate of ADAM was 0.001 and the batch size was set to 128 .

\section{Results and Discussion}

\subsection{Experimental Setup}

In this section, we elaborate on the details of our experimental set-ups, outcomes, and the significance of the results. Figure 2 summarizes the overview of the automatic sleep stage classification process using a $\mathrm{CNN}$ model we developed. To evaluate the performance of the proposed CNN architecture, we designed two experiments: (1) training a CNN model that could correctly classify sleep stages and evaluate the performance with the ISRUC dataset, and (2) Using the trained CNN model to classify the sleep stages in the newly measured lab data. These two experiments were conducted on a laptop equipped with an Intel i7 processor $(\mathrm{I} 7-9750 \mathrm{H})$. To compare the transferability, both experiments were performed with two different types of input data: raw data and multi-taper spectrogram data.

In the first experiment, the first 100 subjects' data were selected in subgroup 1 of the ISRUC dataset. To enhance the accuracy and minimize the bias associated with our classification model, only the epochs where the two scorers agreed with each other were used. The epochs were then split into three parts: $60 \%$ of the dataset for training $(42,094$ epochs), $20 \%$ for validation (14,032 epochs), and $20 \%$ for the test (14,032 epochs). The number of epochs of each of the five classes for the three separate parts is listed in Table 1. For each training step, the weight of the $\mathrm{CNN}$ network parameters was updated based on the result of model training validation accuracy. Most of the hyperparameter values (learning rate, kernel size, and filter of each convolutional layer, and unit of each dropout) were selected by a random search method. In the end, we chose the model with the highest validation accuracy as our best model. The performance of this best model was evaluated based on the prediction accuracy of the test dataset. 
Table 1. Number of epochs of each sleep stage for training, validation, and testing.

\begin{tabular}{|c|c|c|c|c|c|c|c|c|c|c|c|c|c|c|c|}
\hline \multirow{3}{*}{ Input Type } & \multicolumn{15}{|c|}{ Number of Epochs (ISRUC Public Dataset) } \\
\hline & \multicolumn{5}{|c|}{ Training Set } & \multicolumn{5}{|c|}{ Validation Set } & \multicolumn{5}{|c|}{ Test Set } \\
\hline & Aw & N1 & N2 & N3 & $\mathbf{R}$ & Aw & N1 & N2 & N3 & $\mathbf{R}$ & Aw & N1 & N2 & N3 & $\mathbf{R}$ \\
\hline Raw signal & 10,968 & 3299 & 13,366 & 8264 & 6197 & 3591 & 1148 & 4570 & 2692 & 2031 & 3617 & 1065 & 4492 & 2739 & 2119 \\
\hline Spectrogram & 11,013 & 3275 & 13,210 & 8189 & 6207 & 3568 & 1073 & 4729 & 2675 & 1987 & 3575 & 1144 & 4469 & 2791 & 2053 \\
\hline
\end{tabular}

As a result, for the multi-taper spectrogram, $\mathrm{CNN}$ architecture, a $(2,2)$ pool size of 2Dmax pooling, 64 filters and a $(3,3)$ kernel size were used on the first two 2D-convolutional layers and 32 filters and a $(3,3)$ kernel size were used on the last 2D-convolutional layers. Moreover, in order, dense layers with the units 1024, 512 and 5 were used for the fullyconnected layer. Batch normalization, first dropout (0.25), and second dropout $(0.40)$ were utilized to prevent overfitting. For the raw-signal CNN + LSTM architecture, four pool sizes of 1D-max pooling, 80 filters, and three kernel sizes were used on the first 1D convolutional layers, and 32 filters and five kernel sizes were used on the last 1D-convolutional layers and unit 10 on the LSTM layers. Moreover, in order, dense layers with the units 64 and 5 were used for the fully-connected layer. Batch normalization and dropout (0.45) were utilized to prevent overfitting (Figure 2C,D).

In the second experiment, we evaluated the transferability of our trained CNN model on our lab dataset. We used the same model architecture as the first experiment. Our best model was used to predict sleep stages in the lab dataset, and this prediction performance was evaluated through comparison with the manual sleep stage scorings performed, based on AASM criteria, by one human expert with about one year of experience.

\subsection{Performance Comparison with Other Works}

The classification model was well-trained, based on the accuracy and loss of the training and validation graphs shown in Figure 3A,B. Table 2 summarizes the result of the first experiment. As a result of the first experiment with multi-taper spectrogram input data, our classification model's prediction of the 100 subjects showed $88.85 \%$ accuracy and a Cohen's kappa value of 0.854 with the consensus scores of the two expert scorers of the ISRUC dataset (Figure 3C). The classification results with raw data as input showed an accuracy of $87.05 \%$ and a Cohen's kappa value of 0.829 .

Table 3 summarizes the result of the second experiment and enumerates the number of epochs of each sleep stage that were included in the lab dataset. As a result of the second experiment with the multi-taper spectrogram input data, an accuracy of $81.85 \%$ and a Cohen's kappa value of 0.734 could be achieved when the exact same classification model was used to predict the lab dataset measured with our own system (Figure 3D). The classification results with raw data as input showed an accuracy of $72.94 \%$ and a Cohen's kappa value of 0.608 . The results of these experiments clearly show that, compared to using just raw signal, converting the signal to multi-taper spectrogram as the input data provides not only comparable or higher classification performance within the public dataset but also superior transferability of the trained model for the classification of another dataset. The average inter-scorer agreement on standard PSG data was usually reported between $82 \%$ and $89 \%$ [3]. In agreement with this reported value, the average agreement between the two expert scorers of the ISRUC dataset was calculated to be $82.00 \%$, with a Cohen's kappa value of 0.766 . The results of the second experiment $(81.85 \%)$ fall very close to this expected inter-scorer reliability value, and this can show the potential of the effective transferability of our classification model into our lab-based custom system with high performance. The hypnograms in Figure 3E,F show the prediction results from the ISRUC and lab datasets, respectively. 


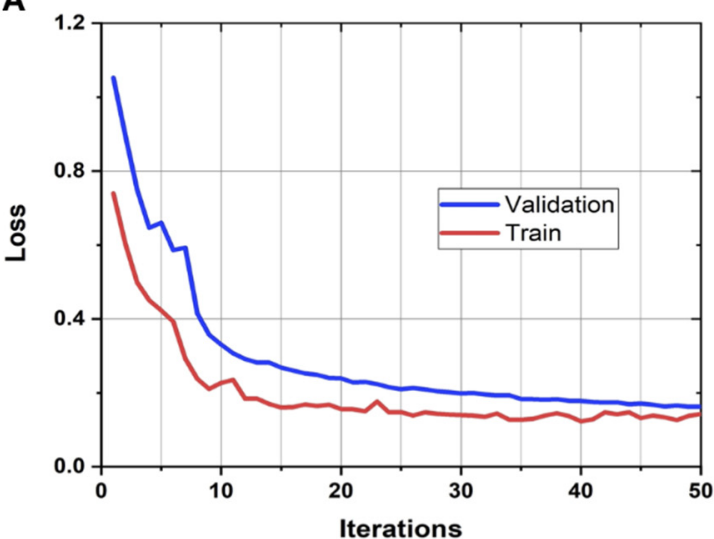

C

ISRUC Dataset Test Accuracy

(Accuracy: $88.85 \%$, Cohen's Kappa $=0.854$ )

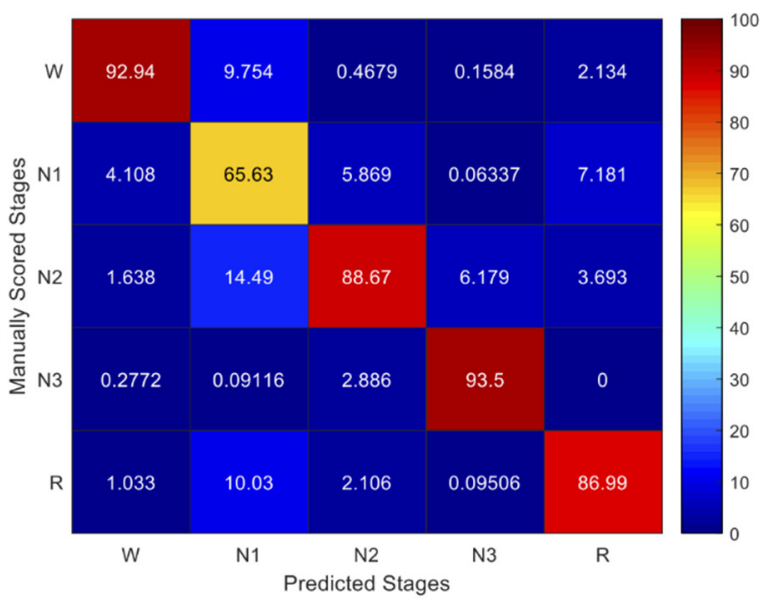

$\mathbf{E}$

Testing Performance

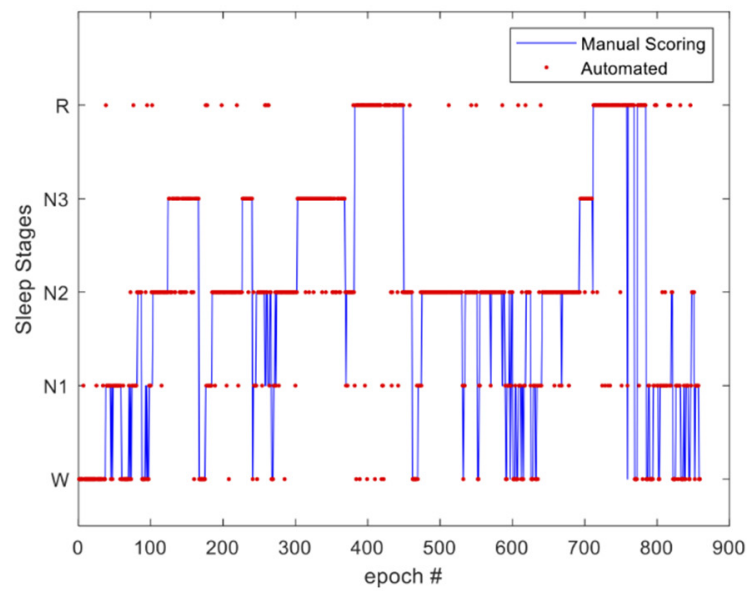

B

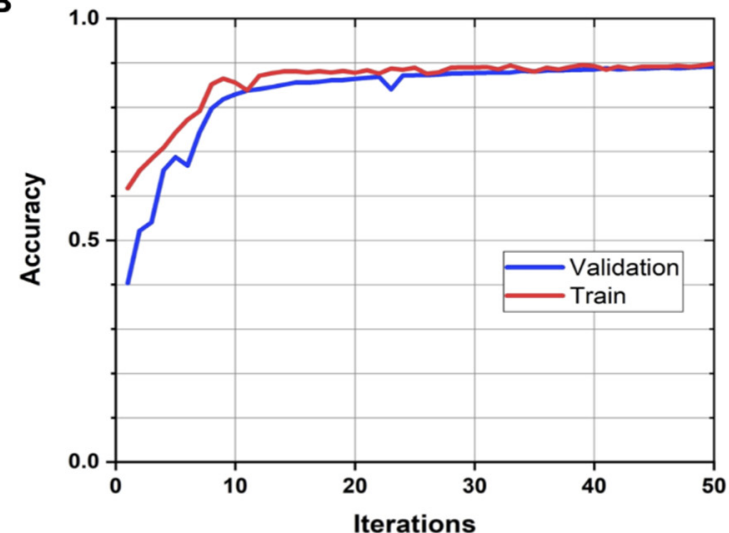

D

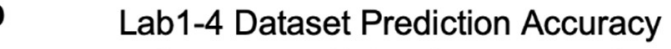

(Accuracy:81.52\%, Cohen's Kappa $=0.734$ )

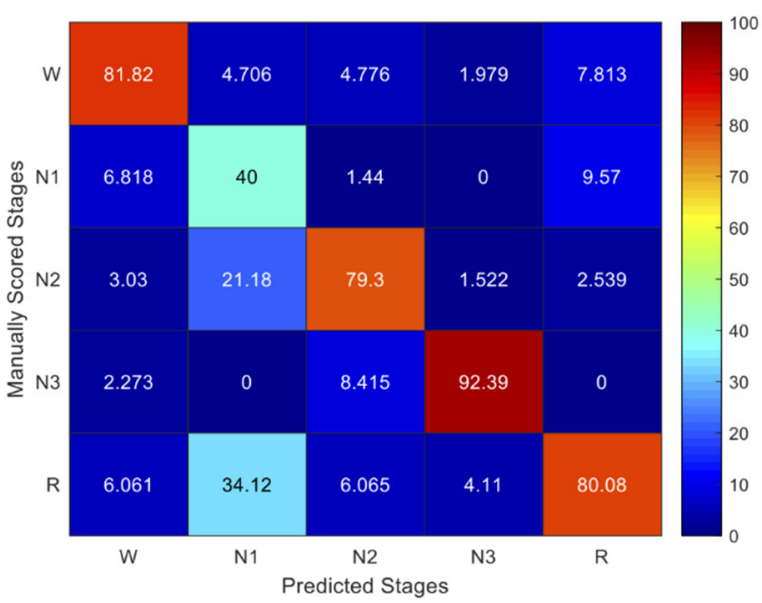

$\mathbf{F}$

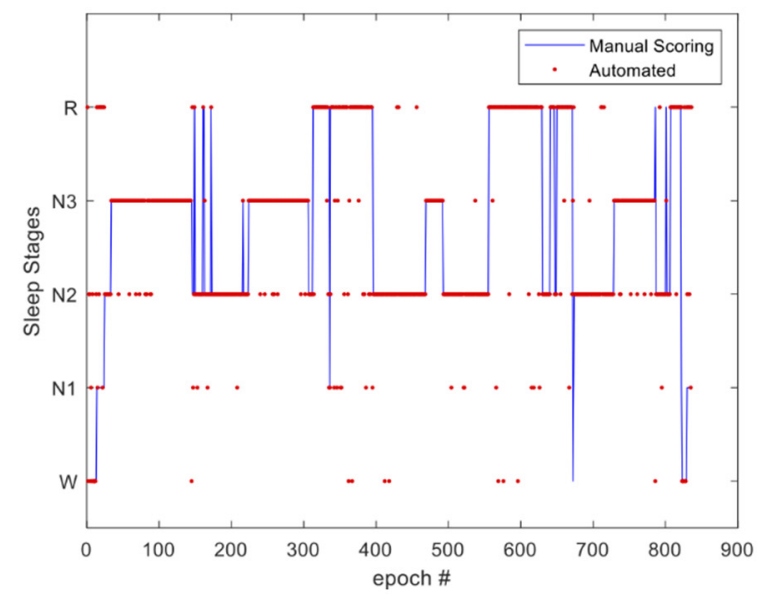

Figure 3. CNN classification results. (A) Loss curves of the training on the ISRUC dataset (red: loss on the training; blue: loss on the testing). (B) Accuracy curves of the training on the ISRUC dataset (red: accuracy on the training; blue: accuracy on the testing). (C) Confusion matrix with the public dataset (accuracy 88.85\%, Cohen's kappa $=0.854$ ). (D) Confusion matrix with lab dataset (accuracy $81.52 \%$, Cohen's kappa $=0.734) .(\mathbf{E}, \mathbf{F})$ Hypnograms of prediction results from the ISRUC $(\mathbf{E})$ and lab datasets (F). 
Table 2. Public dataset based on classification models trained and tested with raw signals and multi-taper spectrograms.

\begin{tabular}{ccc}
\hline \multirow{2}{*}{ Input Type } & \multicolumn{2}{c}{ ISRUC Public Dataset } \\
\cline { 2 - 3 } & Test Accuracy & Cohen's Kappa \\
\hline Raw signal & $87.05 \%$ & 0.829 \\
Multi-taper spectrogram & $88.85 \%$ & 0.854 \\
\hline
\end{tabular}

Table 3. Lab dataset prediction based on classification models trained and tested with raw signal and multi-taper spectrograms, and the combined number of epochs of each sleep stage.

\begin{tabular}{cccccccc}
\hline & \multicolumn{2}{c}{ Lab Dataset } & \multicolumn{3}{c}{ Number of Epochs (Lab Dataset) } \\
\cline { 2 - 7 } Input Type & \multirow{2}{*}{ Prediction Accuracy } & Cohen's Kappa & & \multicolumn{4}{c}{ Prediction Set } \\
\cline { 3 - 7 } & & & Aw & N1 & N2 & N3 & R \\
\hline Raw signal & $72.94 \%$ & 0.608 & 230 & 111 & 1091 & 721 & 554 \\
Multi-taper spectrogram & $81.52 \%$ & 0.734 & 230 & 111 & 1091 & 721 & 554 \\
\hline
\end{tabular}

Table 4 compares the performance of our CNN method in sleep stage classification with other prior works. To evaluate the performance of the sleep stage classification, there are multiple performance metrics being used in the field, including sensitivity, specificity, and F-measure. Among these metrics, the accuracy rate and Cohen's kappa coefficient are the most commonly used metrics [3], so these metrics are presented and used for comparison in this table. Most of the existing works focused on analyzing public sleep datasets, except for a few cases. Compared to the resulting accuracy and kappa values of these works, our work in the first experiment within the public dataset shows comparable performance. Among the prior works, the work from Bresch et al. presented a study similar to ours where a classification model was built from a public dataset (SIESTA) and transfer-tested to a private dataset [30]. Their results showed a kappa value of 0.760 in the public dataset and 0.703 in their private dataset. Compared to this work, our work shows improved performance with both the public and private datasets. Overall, this study shows the human-level performance of our $\mathrm{CNN}$-based sleep classification model in scoring the standard PSG dataset and presents the potential of its effective transferability to other types of datasets, such as our own custom lab dataset with novel nanomembrane electrodes.

Although we could demonstrate transferability of the classification model from the public PSG dataset to a private dataset, the setup and results from the current study still possess some limitations that need to be considered for future studies. First, the public dataset used for this study, ISRUC subgroup 1, includes data from subjects with sleep disorders. Sleep patterns and signal characteristics of subjects with various sleep disorders are likely to be different from those of healthy subjects, and this aspect could have led to reduced performance when the classification model was applied to healthy subject data. Since this study was intended to build a model to classify data from healthy subjects, for future studies, the inclusion of data from a healthy population will be necessary and helpful to enhance the classification performance. Next, a small population of four subjects was used for the tested lab dataset to explore the potential of the transferability of the classification model and to compare the pre-processing methods for more effective transferability. To further validate the effectiveness of multi-taper spectrograms along with a CNN to build a more globally transferable classification model, a much larger number of subjects from various cohorts will need to be included in future studies. 
Table 4. Comparison of sleep-stage classification performance with prior works.

\begin{tabular}{|c|c|c|c|c|c|c|c|c|}
\hline Ref. & Year & Data Type & Input Data & $\begin{array}{c}\text { Number of } \\
\text { Subjects }\end{array}$ & $\begin{array}{c}\begin{array}{c}\text { Public } \\
\text { Dataset }\end{array} \\
\begin{array}{c}\text { Accuracy } \\
(\%) \\
\text { /Kappa }\end{array}\end{array}$ & $\begin{array}{c}\text { Private } \\
\text { Dataset } \\
\begin{array}{c}\text { Accuracy } \\
(\%) \\
\text { /Kappa }\end{array}\end{array}$ & $\begin{array}{l}\text { Number of } \\
\text { Channels }\end{array}$ & $\begin{array}{l}\text { Classification } \\
\text { Method }\end{array}$ \\
\hline $\begin{array}{l}\text { This } \\
\text { work }\end{array}$ & 2022 & $\begin{array}{l}\text { ISRUC and } \\
\text { Lab dataset }\end{array}$ & $\begin{array}{l}\text { Multi-taper } \\
\text { spectrogram } \\
\text { and } \\
\text { Raw data }\end{array}$ & 100 & $\begin{array}{l}88.85 / 0.854 \\
87.05 / 0.829\end{array}$ & $\begin{array}{l}81.52 / 0.734 \\
72.94 / 0.608\end{array}$ & $\begin{array}{c}2 \mathrm{EEG}, 2 \\
\text { EOG }\end{array}$ & CNN \\
\hline$[31]$ & 1993 & $\begin{array}{c}\text { Private } \\
\text { data }\end{array}$ & $\begin{array}{l}\text { Extracted } \\
\text { features }\end{array}$ & 12 & - & $80.60 /-$ & $\begin{array}{c}2 \text { EEG, } 1 \\
\text { EOG, } 1 \text { EMG }\end{array}$ & $\begin{array}{l}\text { Multilayer } \\
\text { Neural } \\
\text { Network }\end{array}$ \\
\hline [32] & 2005 & SIESTA & $\begin{array}{l}\text { Extracted } \\
\text { features }\end{array}$ & 590 & $79.6 / 0.72$ & - & $\begin{array}{c}1 \text { EEG, } 2 \\
\text { EOG, } 1 \text { EMG }\end{array}$ & $\begin{array}{l}\text { LDA, Decision } \\
\text { tree }\end{array}$ \\
\hline [5] & 2014 & Sleep-EDF & $\begin{array}{l}\text { Extracted } \\
\text { features }\end{array}$ & 1 & $88.9 /-$ & - & $1 \mathrm{EEG}$ & SVM \\
\hline [33] & 2016 & Sleep-EDF & Raw data & 20 & $74 / 0.65$ & - & $1 \mathrm{EEG}$ & $\mathrm{CNN}$ \\
\hline$[34]$ & 2016 & Sleep-EDF & $\begin{array}{l}\text { Extracted } \\
\text { features }\end{array}$ & 20 & $78 /-$ & - & $1 \mathrm{EEG}$ & $\begin{array}{l}\text { Stacked Sparse } \\
\text { Autoencoders }\end{array}$ \\
\hline [35] & 2017 & $\begin{array}{l}\text { Montreal } \\
\text { archive }\end{array}$ & $\begin{array}{l}\text { Extracted } \\
\text { features }\end{array}$ & 62 & $83.35 /-$ & - & $1 \mathrm{EEG}$ & $\begin{array}{l}\text { Mixed Neural } \\
\text { Network }\end{array}$ \\
\hline$[36]$ & 2017 & $\begin{array}{l}\text { Sleep-EDF } \\
\text { \& Montreal }\end{array}$ & Raw data & 32 & $86.2 / 0.80$ & - & $1 \mathrm{EEG}$ & $\begin{array}{l}\text { DeepSleepNET } \\
\text { (CNN + LSTM) }\end{array}$ \\
\hline [37] & 2018 & $\begin{array}{l}\text { Montreal } \\
\text { archive }\end{array}$ & Raw data & 61 & $78 / 0.80$ & - & $\begin{array}{c}6 \text { EEG, } 2 \\
\text { EOG, } 3 \text { EMG }\end{array}$ & $\begin{array}{l}\text { Multivariate } \\
\text { Network }\end{array}$ \\
\hline$[38]$ & 2018 & $\begin{array}{l}\text { Private } \\
\text { dataset }\end{array}$ & $\begin{array}{l}\text { Extracted } \\
\text { features }\end{array}$ & 76 & - & $-/ 0.8$ & $\begin{array}{c}1 \mathrm{EEG}, 2 \\
\mathrm{EOG}\end{array}$ & $\begin{array}{c}\text { Random Forest, } \\
\text { CNN, LSTM }\end{array}$ \\
\hline [39] & 2018 & SHHS & Raw data & 5728 & $87 / 0.81$ & - & $1 \mathrm{EEG}$ & $\mathrm{CNN}$ \\
\hline [40] & 2018 & $\begin{array}{l}12 \text { sleep } \\
\text { centers }\end{array}$ & Raw data & 1086 & $87 / 0.766$ & - & $\begin{array}{c}4 \text { EEG, } 2 \\
\text { EOG, } 1 \text { EMG }\end{array}$ & CNN \\
\hline [7] & 2018 & ISRUC & $\begin{array}{l}\text { Extracted } \\
\text { features }\end{array}$ & 100 & $75.29 /-$ & - & $6 \mathrm{EEG}$ & Random Forest \\
\hline [41] & 2018 & ISRUC & Raw data & 116 & $92.2 /-$ & - & $\begin{array}{c}\text { 6 EEG, } 2 \\
\text { EOG, } 3 \text { EMG }\end{array}$ & CNN \\
\hline$[30]$ & 2018 & $\begin{array}{l}\text { SIESTA/private } \\
\text { data }\end{array}$ & Raw data & 147 & $-/ 0.760$ & $-/ 0.703$ & $1 \underset{\mathrm{EOG}, 2}{\mathrm{EEG}}$ & RNN \\
\hline [6] & 2019 & ISRUC & $\begin{array}{l}\text { Extracted } \\
\text { features }\end{array}$ & 10 & $79.64 / 0.74$ & - & $6 \mathrm{EEG}$ & HMM \\
\hline [42] & 2019 & Sleep-EDF & Raw data & 61 & $91.22 /-$ & - & $1 \underset{\mathrm{EOG}}{1 \mathrm{EEG}} 1$ & $\mathrm{CNN}$ \\
\hline [43] & 2019 & $\begin{array}{l}\text { Montreal } \\
\text { archive }\end{array}$ & $\begin{array}{l}\text { Extracted } \\
\text { features }\end{array}$ & 200 & $83.6 /-$ & - & $\begin{array}{c}1 \mathrm{EEG}, 1 \mathrm{EOG}, \\
1 \mathrm{EMG}\end{array}$ & $\mathrm{CNN}$ \\
\hline$[44]$ & 2020 & ISRUC & $\begin{array}{l}\text { Extracted } \\
\text { features }\end{array}$ & 10 & $81.65 / 0.76$ & - & $1 \mathrm{EEG}$ & IMBEFs \\
\hline [45] & 2020 & Sleep-EDF & Raw data & 100 & $85.52 /-$ & - & 2 EEG & $\mathrm{CNN}$ \\
\hline [46] & 2020 & ISRUC & Raw data & 294 & $81.8 / 0.72$ & - & $\begin{array}{c}2 \text { EEG, } 2 \\
\text { EOG, } 1 \text { EMG, } \\
1 \text { ECG }\end{array}$ & $\mathrm{CNN}+\mathrm{RNN}$ \\
\hline
\end{tabular}

Moreover, despite the transferability of the classification model shown in this study, there was a clear reduction in accuracy when the model was tested on the lab dataset. This reduction in the accuracy came from the intrinsic differences between the two different measurement settings, such as subject population, electrode type, equipment, sampling rate, etc. These discrepancies resulted in slightly different spectral analysis signal characteristics and reduced classification performance. One of the critical differences was the electrode locations, especially the EEG electrodes on the forehead and the common reference electrode on the nose. Although the nose is considered to be a relatively inactive 
area, nose referencing still suffers from larger artifacts from facial muscle activity in both the EEG and EOG channels, and especially EOG artifacts in the EEG channels, due to its proximity to the eyes $[47,48]$ In our lab system, the EEG electrodes were placed closer to the eyes on the forehead, and the reference was placed on the nose. With the measurement and reference electrode of each EEG channel placed on the top and bottom sides of the eyes, the vertical eye movement signals observed in the EEG channels were large, and they were also larger than those observed in our EOG channels. As shown in Figure 1E, the described discrepancy in the signals observed from the EEG and EOG channels of each system could be observed, with slightly different spectral characteristics at each stage potentially leading to a reduction in accuracy, most likely in stages N1, where slow eye movement (SEM) is present, and $\mathrm{R}$, where rapid eye movement (REM) is present. In stages N1 and R of the public dataset, the EOG channels generally showed stronger activity observed in the lower frequency range, caused by SEM and REM, as compared to the EEG channels. On the other hand, in the lab datasets, the EEG channels often show comparable or stronger activity in the lower frequency range compared to the EOG channels, caused by strong vertical eye movement signals in the EEG channels and stronger horizontal eye movement signals observed in the EOG channels. In addition to the well-known difficulty in classifying N1, the number of $\mathrm{N} 1$ epochs used for both training the model and prediction of the lab dataset was much smaller compared to other stages, and this could have resulted in the misrepresentation of the model's classification performance of N1 with the numerical results obtained in this study $[49,50]$. These discrepancies between the systems and the smaller number of channels in our system could have led to less accurate scoring of the lab dataset, as it was not measured with the standard PSG setup used for proper scoring with AASM criteria, which resulted in larger uncertainty in the classification performance assessment. For future study, the use of both a standard setup and our novel lab-based setup is desired for more objective and fair classification performance assessment, comparison with expert scorings and inter-scorer reliability.

Furthermore, there were two factors that could have led to the overestimation of our classification model's performance within the public dataset: (1) using consensus epochs for the testing of the model, and (2) using all subjects' data throughout the training, validation, and testing without subject separation. Consensus epochs have clearer signal characteristics of corresponding sleep stages compared to the epochs without consensus, so using consensus epochs for the testing of model may have led to performance overestimation. For future studies, it would be preferred to use all epochs for performance evaluation, and compare the results with inter-scorer reliability. Moreover, due to signal characteristic variability from subject to subject, a more objective evaluation of the performance would be achieved by separating the epochs of certain subjects and keeping them independent for testing purposes alone. If the same subject's epochs used for training and validation are also used for testing, the testing performance could be higher than the result obtained when the model is used on another subject's data. For future studies, the use of the leave-onesubject-out method for the evaluation of the model would be preferred, for more objective evaluation and comparison with other works.

\section{Conclusions}

In this work, we presented an automatic sleep stage classification model that could achieve good performance on the public dataset and accurately predict the sleep stage on our own laboratory dataset. A set of nanomembrane electrodes and custom wireless circuits were used to record lab datasets with EEG and EOG from multiple subjects during their sleep. We developed a classification model based on CNN, which was utilized for training and validating the classifier model based on the ISRUC public dataset using two EEG (F3-A2, F4-A1) and two EOG (EOGL, EOGR) channels. Then we transferred our model to the classification of our experimental dataset, which was collected with the nanomembrane electrodes. Overall, the collective results show that our model had high performance on both the test dataset (accuracy $=88.85 \%$, Cohen's kappa $=0.854$ ) and on our lab dataset 
(accuracy $=81.85 \%$, Cohen's kappa $=0.734)$. Future work will resolve the limitations of the current study discussed above and expand this research to include a larger group of sleep patients to measure data with the wearable system for automatic sleep stage classification.

Author Contributions: K.K., S.K. and W.-H.Y. conceived and designed the research; K.K. designed the machine learning algorithm; S.K. conducted the experimental study; K.K., S.K. and W.-H.Y. wrote the paper. All authors have read and agreed to the published version of the manuscript.

Funding: We acknowledge the support from the IEN Center for Human-Centric Interfaces and Engineering at Georgia Tech, and this study was partially supported by the Institute of Information and Communications Technology Planning and Evaluation (IITP) grant funded by the Korean government (MSIT) (2021-0-01517). The electronic devices used in this work were fabricated at the Institute for Electronics and Nanotechnology, a member of the National Nanotechnology Coordinated Infrastructure, which is supported by the National Science Foundation (grant ECCS-2025462).

Institutional Review Board Statement: The study was conducted according to the guidelines of the following the approved IRB protocol (\#H20211) at the Georgia Institute of Technology.

Informed Consent Statement: Informed consent was obtained from all subjects involved in the study.

Data Availability Statement: The data presented in this study are available on request from the corresponding author.

Conflicts of Interest: Georgia Tech has a pending US patent application related to the work described here.

\section{References}

1. Rahman, M.M.; Bhuiyan, M.I.H.; Hassan, A.R. Sleep stage classification using single-channel EOG. Comput. Biol. Med. 2018, 102, 211-220. [CrossRef]

2. Kim, H.; Kwon, S.; Kwon, Y.-T.; Yeo, W.-H. Soft Wireless Bioelectronics and Differential Electrodermal Activity for Home Sleep Monitoring. Sensors 2021, 21, 354. [CrossRef] [PubMed]

3. Kwon, S.; Kim, H.; Yeo, W.-H. Recent advances in wearable sensors and portable electronics for sleep monitoring. Iscience 2021, 24, 102461. [CrossRef] [PubMed]

4. Armon, C. Polysomnography. Medscape 2020, 31, 281-297.

5. Zhu, G.; Li, Y.; Wen, P.P. Analysis and classification of sleep stages based on difference visibility graphs from a single-channel EEG signal. IEEE J. Biomed. Health Inform. 2014, 18, 1813-1821. [CrossRef]

6. Ghimatgar, H.; Kazemi, K.; Helfroush, M.S.; Aarabi, A. An automatic single-channel EEG-based sleep stage scoring method based on hidden Markov Model. J. Neurosci. Methods 2019, 324, 108320. [CrossRef]

7. Tzimourta, K.D.; Tsilimbaris, A.; Tzioukalia, K.; Tzallas, A.T.; Tsipouras, M.G.; Astrakas, L.G.; Giannakeas, N. EEG-based automatic sleep stage classification. Biomed. J. 2018, 1, 6.

8. Khalighi, S.; Sousa, T.; Santos, J.M.; Nunes, U. ISRUC-Sleep: A comprehensive public dataset for sleep researchers. Comput. Methods Programs Biomed. 2016, 124, 180-192. [CrossRef]

9. Lim, H.R.; Kim, H.S.; Qazi, R.; Kwon, Y.T.; Jeong, J.W.; Yeo, W.H. Advanced soft materials, sensor integrations, and applications of wearable flexible hybrid electronics in healthcare, energy, and environment. Adv. Mater. 2020, 32, 1901924. [CrossRef]

10. Herbert, R.; Kim, J.-H.; Kim, Y.S.; Lee, H.M.; Yeo, W.-H. Soft material-enabled, flexible hybrid electronics for medicine, healthcare, and human-machine interfaces. Materials 2018, 11, 187. [CrossRef]

11. Kwon, S.; Kwon, Y.-T.; Kim, Y.-S.; Lim, H.-R.; Mahmood, M.; Yeo, W.-H. Skin-conformal, soft material-enabled bioelectronic system with minimized motion artifacts for reliable health and performance monitoring of athletes. Biosens. Bioelectron. 2020, 151, 111981. [CrossRef] [PubMed]

12. Kim, Y.-S.; Mahmood, M.; Kwon, S.; Herbert, R.; Yeo, W.-H. Wireless Stretchable Hybrid Electronics for Smart, Connected, and Ambulatory Monitoring of Human Health. In Proceedings of the Meeting Abstracts; IOP Publishing: Bristol, UK, 2019 ; p. 2293.

13. George, N.; Jemel, B.; Fiori, N.; Renault, B. Face and shape repetition effects in humans: A spatio-temporal ERP study. NeuroReport 1997, 8, 1417-1422. [CrossRef] [PubMed]

14. Teplan, M. Fundamentals of EEG measurement. Meas. Sci. Rev. 2002, 2, 1-11.

15. Yao, D.; Qin, Y.; Hu, S.; Dong, L.; Vega, M.L.B.; Sosa, P.A.V. Which reference should we use for EEG and ERP practice? Brain Topogr. 2019, 32, 530-549. [CrossRef] [PubMed]

16. O'Regan, S.; Faul, S.; Marnane, W. Automatic detection of EEG artefacts arising from head movements. In Proceedings of the 2010 Annual International Conference of the IEEE Engineering in Medicine and Biology, Buenos Aires, Argentina, 31 August-4 September 2010; pp. 6353-6356. 
17. Norton, J.J.; Lee, D.S.; Lee, J.W.; Lee, W.; Kwon, O.; Won, P.; Jung, S.-Y.; Cheng, H.; Jeong, J.-W.; Akce, A. Soft, curved electrode systems capable of integration on the auricle as a persistent brain-computer interface. Proc. Natl. Acad. Sci. USA 2015, 112, 3920-3925. [CrossRef]

18. Mahmood, M.; Mzurikwao, D.; Kim, Y.-S.; Lee, Y.; Mishra, S.; Herbert, R.; Duarte, A.; Ang, C.S.; Yeo, W.-H. Fully portable and wireless universal brain-machine interfaces enabled by flexible scalp electronics and deep learning algorithm. Nat. Mach. Intell. 2019, 1, 412-422. [CrossRef]

19. Tian, L.; Zimmerman, B.; Akhtar, A.; Yu, K.J.; Moore, M.; Wu, J.; Larsen, R.J.; Lee, J.W.; Li, J.; Liu, Y. Large-area MRI-compatible epidermal electronic interfaces for prosthetic control and cognitive monitoring. Nat. Biomed. Eng. 2019, 3, 194-205. [CrossRef]

20. Mahmood, M.; Kwon, S.; Berkmen, G.K.; Kim, Y.-S.; Scorr, L.; Jinnah, H.; Yeo, W.-H. Soft nanomembrane sensors and flexible hybrid bioelectronics for wireless quantification of blepharospasm. IEEE Trans. Biomed. Eng. 2020, 67, 3094-3100. [CrossRef]

21. Zavanelli, N.; Kim, H.; Kim, J.; Herbert, R.; Mahmood, M.; Kim, Y.-S.; Kwon, S.; Bolus, N.B.; Torstrick, F.B.; Lee, C.S. At-home wireless monitoring of acute hemodynamic disturbances to detect sleep apnea and sleep stages via a soft sternal patch. Sci. Adv. 2021, 7, eabl4146. [CrossRef]

22. Kandel, E.R.; Schwartz, J.H.; Jessell, T.M.; Siegelbaum, S.; Hudspeth, A.J.; Mack, S. Principles of Neural Science; McGraw-Hill: New York, NY, USA, 2000; Volume 4.

23. Vrbancic, G.; Podgorelec, V. Automatic classification of motor impairment neural disorders from EEG signals using deep convolutional neural networks. Elektron. Ir Elektrotechnika 2018, 24, 3-7. [CrossRef]

24. Kuanar, S.; Athitsos, V.; Pradhan, N.; Mishra, A.; Rao, K.R. Cognitive analysis of working memory load from EEG, by a deep recurrent neural network. In Proceedings of the 2018 IEEE International Conference on Acoustics, Speech and Signal Processing (ICASSP), Calgary, AB, Canada, 15-20 April 2018; pp. 2576-2580.

25. Vilamala, A.; Madsen, K.H.; Hansen, L.K. Deep convolutional neural networks for interpretable analysis of EEG sleep stage scoring. In Proceedings of the 2017 IEEE 27th international workshop on machine learning for signal processing (MLSP), Tokyo, Japan, 25-28 September 2017; pp. 1-6.

26. Jiao, Z.; Gao, X.; Wang, Y.; Li, J.; Xu, H. Deep convolutional neural networks for mental load classification based on EEG data. Pattern Recognit. 2018, 76, 582-595. [CrossRef]

27. Prerau, M.J.; Brown, R.E.; Bianchi, M.T.; Ellenbogen, J.M.; Purdon, P.L. Sleep neurophysiological dynamics through the lens of multitaper spectral analysis. Physiology 2017, 32, 60-92. [CrossRef] [PubMed]

28. Kwon, Y.-T.; Kim, Y.-S.; Kwon, S.; Mahmood, M.; Lim, H.-R.; Park, S.-W.; Kang, S.-O.; Choi, J.J.; Herbert, R.; Jang, Y.C. All-printed nanomembrane wireless bioelectronics using a biocompatible solderable graphene for multimodal human-machine interfaces. Nat. Commun. 2020, 11, 1-11. [CrossRef] [PubMed]

29. Jeong, J.W.; Yeo, W.H.; Akhtar, A.; Norton, J.J.; Kwack, Y.J.; Li, S.; Jung, S.Y.; Su, Y.; Lee, W.; Xia, J. Materials and optimized designs for human-machine interfaces via epidermal electronics. Adv. Mater. 2013, 25, 6839-6846. [CrossRef] [PubMed]

30. Bresch, E.; Großekathöfer, U.; Garcia-Molina, G. Recurrent deep neural networks for real-time sleep stage classification from single channel EEG. Front. Comput. Neurosci. 2018, 12, 85. [CrossRef]

31. Schaltenbrand, N.; Lengelle, R.; Macher, J.-P. Neural network model: Application to automatic analysis of human sleep. Comput. Biomed. Res. 1993, 26, 157-171. [CrossRef]

32. Anderer, P.; Gruber, G.; Parapatics, S.; Woertz, M.; Miazhynskaia, T.; Klösch, G.; Saletu, B.; Zeitlhofer, J.; Barbanoj, M.J.; DankerHopfe, H. An E-health solution for automatic sleep classification according to Rechtschaffen and Kales: Validation study of the Somnolyzer $24 \times 7$ utilizing the Siesta database. Neuropsychobiology 2005, 51, 115-133. [CrossRef]

33. Tsinalis, O.; Matthews, P.M.; Guo, Y.; Zafeiriou, S. Automatic sleep stage scoring with single-channel EEG using convolutional neural networks. arXiv 2016, arXiv:1610.01683.

34. Tsinalis, O.; Matthews, P.M.; Guo, Y. Automatic sleep stage scoring using time-frequency analysis and stacked sparse autoencoders. Ann. Biomed. Eng. 2016, 44, 1587-1597. [CrossRef]

35. Dong, H.; Supratak, A.; Pan, W.; Wu, C.; Matthews, P.M.; Guo, Y. Mixed neural network approach for temporal sleep stage classification. IEEE Trans. Neural Syst. Rehabil. Eng. 2017, 26, 324-333. [CrossRef]

36. Supratak, A.; Dong, H.; Wu, C.; Guo, Y. DeepSleepNet: A model for automatic sleep stage scoring based on raw single-channel EEG. IEEE Trans. Neural Syst. Rehabil. Eng. 2017, 25, 1998-2008. [CrossRef] [PubMed]

37. Chambon, S.; Galtier, M.N.; Arnal, P.J.; Wainrib, G.; Gramfort, A. A deep learning architecture for temporal sleep stage classification using multivariate and multimodal time series. IEEE Trans. Neural Syst. Rehabil. Eng. 2018, 26, 758-769. [CrossRef] [PubMed]

38. Malafeev, A.; Laptev, D.; Bauer, S.; Omlin, X.; Wierzbicka, A.; Wichniak, A.; Jernajczyk, W.; Riener, R.; Buhmann, J.; Achermann, P. Automatic human sleep stage scoring using deep neural networks. Front. Neurosci. 2018, 12, 781. [CrossRef] [PubMed]

39. Sors, A.; Bonnet, S.; Mirek, S.; Vercueil, L.; Payen, J.-F. A convolutional neural network for sleep stage scoring from raw single-channel EEG. Biomed. Signal Process. Control 2018, 42, 107-114. [CrossRef]

40. Stephansen, J.B.; Olesen, A.N.; Olsen, M.; Ambati, A.; Leary, E.B.; Moore, H.E.; Carrillo, O.; Lin, L.; Han, F.; Yan, H. Neural network analysis of sleep stages enables efficient diagnosis of narcolepsy. Nat. Commun. 2018, 9, 1-15. [CrossRef]

41. Cui, Z.; Zheng, X.; Shao, X.; Cui, L. Automatic sleep stage classification based on convolutional neural network and fine-grained segments. Complexity 2018, 2018. [CrossRef] 
42. Yildirim, O.; Baloglu, U.B.; Acharya, U.R. A deep learning model for automated sleep stages classification using PSG signals. Int. J. Environ. Res. Public Health 2019, 16, 599. [CrossRef]

43. Phan, H.; Andreotti, F.; Cooray, N.; Chén, O.Y.; De Vos, M. Joint classification and prediction CNN framework for automatic sleep stage classification. IEEE Trans. Biomed. Eng. 2018, 66, 1285-1296. [CrossRef]

44. Shen, H.; Ran, F.; Xu, M.; Guez, A.; Li, A.; Guo, A. An Automatic Sleep Stage Classification Algorithm Using Improved Model Based Essence Features. Sensors 2020, 20, 4677. [CrossRef]

45. Lee, T.; Hwang, J.; Lee, H. Trier: Template-guided neural networks for robust and interpretable sleep stage identification from eeg recordings. arXiv 2020, arXiv:2009.05407.

46. Zhang, X.; Xu, M.; Li, Y.; Su, M.; Xu, Z.; Wang, C.; Kang, D.; Li, H.; Mu, X.; Ding, X. Automated multi-model deep neural network for sleep stage scoring with unfiltered clinical data. Sleep Breath. 2020, 24, 581-590. [CrossRef] [PubMed]

47. Essl, M.; Rappelsberger, P. EEG cohererence and reference signals: Experimental results and mathematical explanations. Med. Biol. Eng. Comput. 1998, 36, 399-406. [CrossRef] [PubMed]

48. Trujillo, L.T.; Peterson, M.A.; Kaszniak, A.W.; Allen, J.J. EEG phase synchrony differences across visual perception conditions may depend on recording and analysis methods. Clin. Neurophysiol. 2005, 116, 172-189. [CrossRef] [PubMed]

49. Mousavi, S.; Afghah, F.; Acharya, U.R. SleepEEGNet: Automated sleep stage scoring with sequence to sequence deep learning approach. PLoS ONE 2019, 14, e0216456. [CrossRef] [PubMed]

50. Melek, M.; Manshouri, N.; Kayikcioglu, T. An automatic EEG-based sleep staging system with introducing NAoSP and NAoGP as new metrics for sleep staging systems. Cogn. Neurodynamics 2021, 15, 405-423. [CrossRef] 